EPJ Web of Conferences 47, 09003 (2013)

DOI: $10.1051 /$ epjconf/20134709003

(C) Owned by the authors, published by EDP Sciences, 2013

\title{
New procedure of the fine analysis of spectra of F-G-K stars
}

\author{
Yakiv V. Pavlenko ${ }^{1,2, a}$ \\ ${ }^{1}$ Main Astronomical Observatory of the National Academy of Sciences \\ ${ }^{2}$ University of Hertfordshire
}

\begin{abstract}
Some problems of the determination of the microturbulent velocities and abundances in atmospheres of the F-G-K stars and determination of their effective temperatures and rotational velocities in the framework of self-consistent approach are discussed. Firstly, we use independent determinations of iron abundances. using the fits to the observed profiles of Fe I and Fe II absorption lines. Secondly, abundances of other elements are determined for the found input parameters.
\end{abstract}

\section{INTRODUCTION}

Recent progress and advance of astrophysical hardware, especially high resolution echelle spectrographs and sophisticated algorithms of the reduction of the experimental data the study push us to the high precision investigation of the essential physical parameters of atmospheres of solar-like stars. From other hand to model their spectra some sophisticated procedures and algorithms of the "fine analysis" pf observed spectra were developed (see [16], [8], [9]). Here and hereafter we use the terminology "the fine analysis" to define a special kind of the investigations based on the comparison of observed and computed profiles of spectral lines in stellar spectra. Here we note the remarkable progress in obtaining more precise basic physical parameters from the latest evolutionary models (see [7], [1], [13]) . Instead of the pure academic interest of the detailed studies of solar-like stars, these investigations were triggered mainly by the recent discovery that the metal content of planet-hosting stars is an important ingredient that seems to affect the formation and evolution of planetary systems (see [14], [15], and references therein). Indeed, detailed studies of the hosting star can attain benchmark status by detailed study of the properties of the primary, and then evolutionary and bulk characteristics can be inferred for the companion. In some cases only in that way we can obtain the information about the status of companions, whether they are brown dwarf or planetary.

\section{OBSERVED DATA}

For simplicity we restrict our consideration be the case of high resolution spectra obtained by the hi-res spectrographs of FEROS or HARPS classes. The observed spectra are reduced by the existing systems of astrophysical software. In fact, these reduced spectra do not have any dopler shifts, the wavelengths are given in the referenced "co-solar" frame. It is worth noting, that the most known automatic procedures of the spectra reduction may provide some false counts as it seen on the left panel of Fig. 1. After the additional reduction spectra of the most solar-like stars look very similar to the solar spectrum (see the left panel of Fig. 1). It is worth noting that the differences seen on the right panel may be explained by

\footnotetext{
ae-mail: yp@mao.kiev.ua
}

This is an Open Access article distributed under the terms of the Creative Commons Attribution License 2.0, which permits unrestricted use, distribution, and reproduction in any medium, provided the original work is properly cited. 

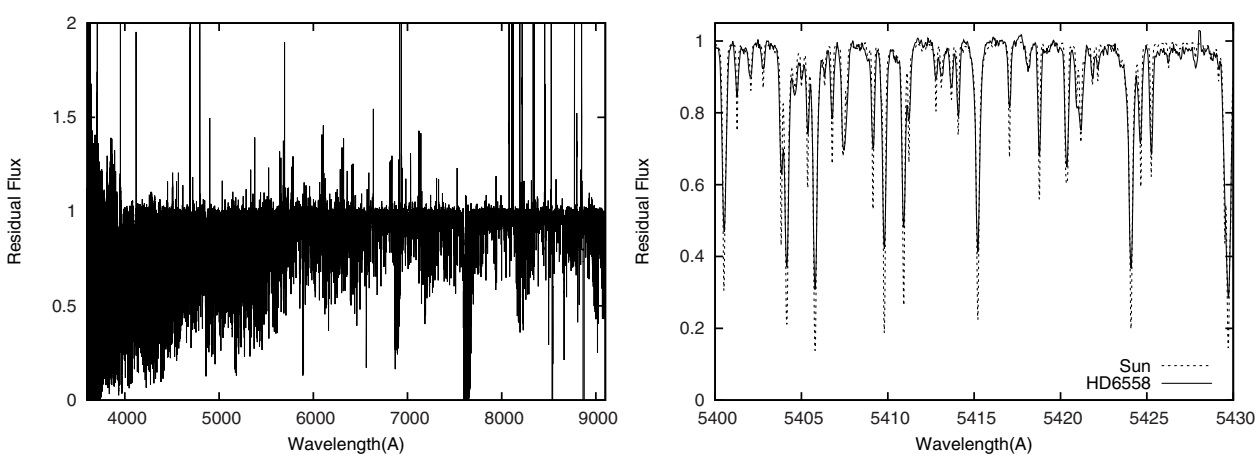

Figure 1. Left: observed FEROS spectrum of HD6558 after the automatic procedure reduction (see [3]). Right: comparison of observed spectra of the Sun as a star [6], and HD6558 [3].
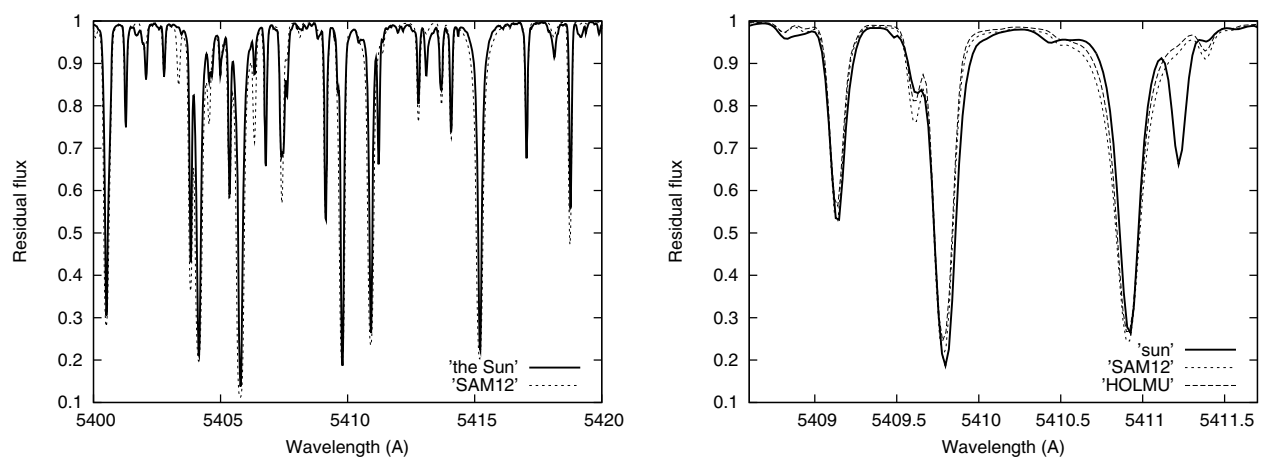

Figure 2. Left: comparison of computed and observed spectrum of the Sun as a star. Right: another comparison in the larger scale.

the cumulative effects of the different effective temperatures, gravities, metallicities and mictorturbulent velocities.

In general, the presence of dopler shifts in the observed spectra is not critical issue for our procedure.

\section{PROCEDURE}

We determine effective temperatures, rotational velocities, microturbulent velocities, and chemical abundances in the atmospheres of the solar-like stars in the framework of self-consistent approach:

- model atmospheres and synthetic spectra are computed by SAM12 [11] and WITA [10] programs, respectively, for the same input data set, i.e. effective temperature, gravity, microturbulent velocity, abundances. In our work we used as well the semiempirical 1D model atmosphere of solar atmosphere HOLMU [2]. Synthetic spectra computed for the SAM12 and HOLMU model atmospheres of the Sun show rather marginal differences shown on the left panel of Fig. 2. More critical for our analysis is the possible errors in the the input line lists (see the right panel of Fig 2). Even in the well studied green part of the solar spectrum we see the presence of some lines with wrong oscillator strength $g f$ (or excitation potential of the lower level $E^{\prime \prime}$ ).

- The spectrum of the Sun is used as the template spectrum to select spectral lines with properly fitted profiles for all elements of our interest.

- For our star we perform our analysis for the the preselected lines of elements of our interest. Blending effects are accounted explicitly in all cases. 

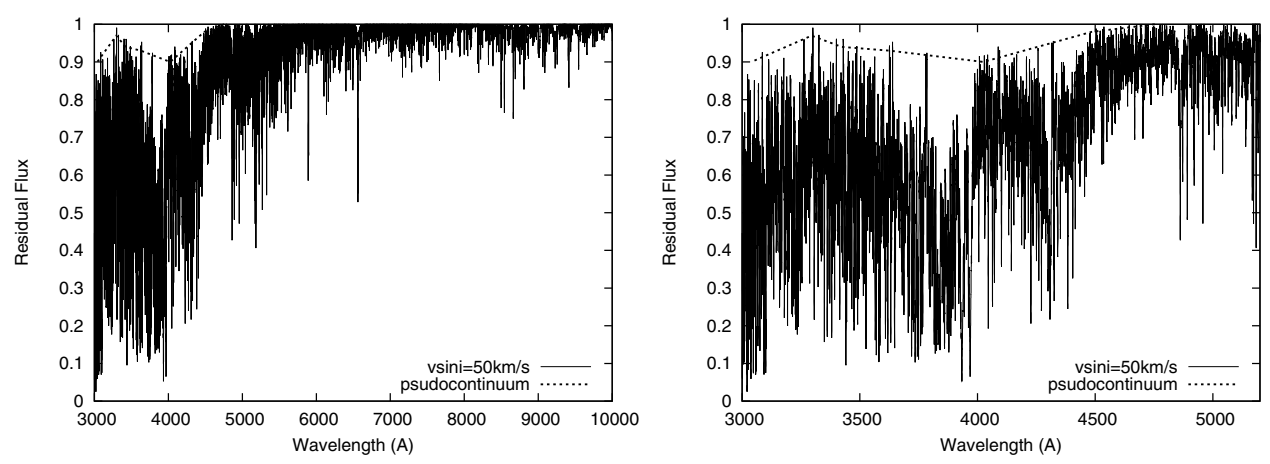

Figure 3. Impact of rotation on the pseudocontinuum level in the theoretical spectrum of the solar-like dwarf. spectra are computed for 5777/4.44/0 model atmosphere and $v \sin i=20 \mathrm{~km} / \mathrm{s}$.

- All parameters are determined by one ABEL procedure. Details of of the procedure are described in Pavlenko et al. [12].

Firstly, we choose the best solution for a set of adopted microturbulent velocities from the fits to these spectral features for the model atmosphere that provides the best confidence in the determined $\log \mathrm{N}(\mathrm{Fe})$, $V_{\mathrm{t}}$, and $v \sin i$. To determine the most self-consistent effective temperature and microturbulent velocity in any star's atmosphere, we used an additional constraint where we minimise the dependence of the derived abundances of $\mathrm{Fe} \mathrm{I}$ and $\mathrm{Fe}$ II on the excitation potential of the corresponding lines.

Secondly, abundances of other elements are determined for the found input parameters.

Procedure is repeated till the final convergence.

\section{ROTATION AND ABUNDANCE ANALYSIS}

Any rotation of the star provides some problems in the analysis of its spectrum. We cannot find any non-blended line in the spectrum of solar-like star with rotation velocity $v \sin i=20 \mathrm{~km} / \mathrm{s}$ and more. Strong blending affects the spectrum in two ways:

a) lines of different species form the common blends. Their analysis seem to be very difficult (see Jenkins et al. 2012).

b) Blended lines in the blue part of the spectrum form the pseudocontinuum, the real continuum if not visible here, like in M-dwarf spectra. In fact, we can apply the procedures based on the ideology of residual intensities only in spectral regions at $\lambda>5200 \AA$ (see Fig. 3).

\section{FIRST RESULTS}

The procedure was used by Pavlenko et al. [12] to analyse the spectra of the Sun and two well known solar type stars, HD1835 and HD10700 to determine their abundances, microturbulent velocity and rotational velocity. We determined self-consistent values for the effective temperatures, abundances, $V_{\mathrm{t}}$ and $v \sin i$. For the Sun we obtain the best agreement for a model atmosphere of $T_{\text {eff }} / \log \mathrm{g} /[\mathrm{Fe} / \mathrm{H}]=$ $5777 / 4.44 / 0.0$, iron abundances and microturbulent velocities of $\log \mathrm{N}(\mathrm{Fe})=4.44, V_{\mathrm{t}}=0.75 \mathrm{~km} / \mathrm{s}$, for the Fe I lines, and $\log \mathrm{N}(\mathrm{Fe})=-4.47, V_{\mathrm{t}}=1.5 \mathrm{~km} / \mathrm{s}$ for the $\mathrm{Fe}$ II lines. Abundances of $\mathrm{Mg}, \mathrm{Ni}$, $\mathrm{Ca}, \mathrm{Ti}$ and $\mathrm{Si}$ obtained from the fits of their absorption features agree well enough $( \pm 0.1 \mathrm{dex})$ with the known values for the Sun. Furthermore we determined a rotational velocity of $v \sin i=1.6 \pm 0.3 \mathrm{~km} / \mathrm{s}$ for the spectrum of the Sun as a star. For HD1835 the self-consistent solution for Fe I and Fe II lines log $\mathrm{N}(\mathrm{Fe})=+0.2$ was obtained with a model atmosphere of 5807/4.47/ +0.2 and microturbulent velocity $V_{\mathrm{t}}=0.75 \mathrm{~km} / \mathrm{s}$, and leads to $v \sin i=7.2 \pm 0.5 \mathrm{~km} / \mathrm{s}$. For HD10700 the self-consistent solution log 


\section{EPJ Web of Conferences}

$\mathrm{N}(\mathrm{Fe})=-4.93$ was obtained using a model atmosphere of 5383/4.59/-0.6 and microturbulent velocity $V_{\mathrm{t}}=0.5 \mathrm{~km} / \mathrm{s}$.

Using the ABEL program Jenkins et al. [4] provide the abundance measurements for the pre-main sequence star PZ Tel recently found to host a young and low-mass companion. Our analysis shows that PZ Tel is fast rotating star, we obtained $v \sin i=73 \pm 5 \mathrm{kms}^{-1}$. That fast rotation provides some problems for our fine analysis procedure (see section 3) but from the fits to a few selected spectral features formed mainly by strong Fe I lines we determined approximately solar metallicity of the star $(\log N(\mathrm{Fe})=-4.37 \mathrm{dex}$ or $[\mathrm{Fe} / \mathrm{H}]=0.05 \pm 0.1 \mathrm{dex})$.

In general, the formal errors of our abundance determination in most cases do not exceed 0.1 dex, a value that is good enough to determine abundances in the atmospheres of a large number of stars and perform statistical analyses for such samples. The work is now in the progress.

I thank James Jenkins, Hugh Jones, Olexij Ivanyuk and David Pinfield for the fruitful collaboration. The computations were carried out on the computer cluster of University of. Hertfordshire. The work was partially supported by the EU PF7 Marie Curie Initial Training Networks (ITN) ROPACS project (GA N 213646) and "Cosmomicrophysics" program of the Academy of. Sciences of Ukraine.

\section{References}

[1] Ghezzi L., Cunha K., Smith V. V., de Araújo F. X., Schuler S. C., de la Reza R., ApJ, 720, 1290 (2010)

[2] Holweger H., Muller E. A., 1974, Solar Phys., 39, 19 (1974)

[3] Jenkins J. S., Jones H. R. A., Pavlenko Ya. V., Pinfield D. J., Barnes J. R., Lyubchik Yu., A\&A, 485, 571 (2008)

[4] Jenkins, J. S., Pavlenko, Y. V., Ivanyuk, O., Gallardo, J., Jones, M. I., Day-Jones, A. C., Jones, H. R. A., Ruiz, M. T., Pinfield, D. J., Yakovina, L. MNRAS, 420, 3587 (2012)

[5] Kupka F., Piskunov N., Ryabchikova T. A., Stempels H. C., Weiss W. W., 1999, A\&AS, 138, 119 (1999)

[6] Kurucz R. L., Furenlid I., Brault J., Testerman L., National Solar. Observatory Atlas, 198 (1984)

[7] Lopez-Santiago J., Montes D., Gálvez-Ortiz M. C., Crespo-Chacón I., Martínez-Arnáiz R. M., Fernández-Figueroa M. J., de Castro E., Cornide M., 2010, A\&A, 514, 97 (2010)

[8] Neves V., Santos N. C., Sousa S. G., Correia A. C. M., Israelian G., A\&A, 497, 563 (2009)

[9] Mashonkina L., Gehren T., Shi J.-R., Korn A.J., Grupp F., A\&A, A87 (2011)

[10] Pavlenko Ya. V., A\&AS, 253, 43 (1997)

[11] Pavlenko Ya. V., Astron. Rept., 47, 59 (2003)

[12] Pavlenko, Ya. V., Jenkins, J. S., Jones, H. R. A., Ivanyuk, O., MNRAS, 422, 542 (2012)

[13] Takeda Y., Honda S., Kawanomoto S., Ando H., Sakurai T., A\&A, 515, 93 (2010)

[14] Santos N. C. et al., A\&A, 526A, 112 (2011)

[15] Sousa S. G., Santos N. C., Israelian G., Mayor M., Udry S., A\&A, 533A, 141 (2011)

[16] Valenti J., Fischer D., ApJS, 159, 141 (2005) 